Retracted Paper

\title{
Elevated Expression of Zinc Finger Protein 703 Promotes Cell Proliferation and Metastasis through PI3K/AKT/GSK- $3 \beta$ Signalling in Oral Squamous Cell Carcinoma
}

\author{
Hao Wang ${ }^{\text {a }}$ Xubin Deng ${ }^{d} \quad$ Jinshan Zhang ${ }^{a}$ Zhilin Ou ${ }^{a} \quad$ Jiajie Maia \\ Shangwei Ding ${ }^{c}$ Shaofen Huo ${ }^{b}$ \\ aDepartment of Radiation Oncology, The Third Affiliated Hospital of Guangzhou Medical University, \\ Guangzhou, bepartment of otorhinolaryngology of Nanfang Hospital, Southern Medical University, \\ Guangzhou, 'Department of Ultrasound, Dongguan People's Hospital Affiliated to Southern Medical \\ University, Dongguan, Guangdong, 'Department of Medical Oncology,Affiliated Cancer Hospital of \\ Guangzhou Medical University, Guangzhou, PR China
}

